\title{
On Structural Accounts of Model-Explanations
}

\section{Introduction}

The focus in the literature on scientific explanation has shifted in recent years towards modelbased approaches. The idea that there are simple and true laws of nature has met with objections from philosophers such as Nancy Cartwright (1983) and Paul Teller (2001), and this has made a strictly Hempelian D-N style explanation largely irrelevant to the explanatory practices of science (Hempel \& Oppenheim, 1948). Much of scientific practice does not involve subsuming particular events under laws of nature. It is increasingly recognized that science across the disciplines is to some degree a patchwork of scientific models, with different methods, strategies, and with varying degrees of success in prediction and explanation. And so accounts of scientific explanation have reflected this change of perspective and model-based approaches have flourished in the explanation literature (Batterman, 2002b; Bokulich, 2008; Craver, 2006; Strevens, 2008; Woodward, 2003). Of course, not all scientific models are explanatory. Some models, especially highly-idealized ones, are merely calculational tools, whose use in the practice of science is entirely predictive or heuristic. Historically, philosophical accounts of scientific explanation have focused on articulating independent criteria for what counts as an explanation.

In recent work, Alisa Bokulich has argued that idealization has a central role to play in explanation (Bokulich, 2008, 2011, 2012). Bokulich claims that certain highly-idealized models are explanatory, even though they are not considered explanatory by causal, mechanistic, or covering law accounts of explanation. She calls these kinds of explanations structural model explanations and argues that the structural similarity between the model and the target system 
can play the role of determining whether or not that model is explanatory (Bokulich, 2008, p. 145). She formulates her account as structural in part to capture models that are not explanatory on, for instance, Woodward's manipulationist account (Woodward, 2003). She aims to expand the store of explanatory models to include as explanatory those that do not accurately represent those that model a physical system by means of fictitious entities or processes, what she calls explanatory fictions.

This paper examines Bokulich's account to assess the success of her formulation of the structural criterion, and also to make the more general claim that there are problems with maintaining that a structural criterion can capture the way that highly-idealized models explain. In order to argue this, I will first examine Bokulich's account as given in (Bokulich, 2008, 2011, 2012). This second section will also employ this account in explaining the phenomenon of quantum wavefunction scarring, which is a pattern in probability distribution in certain quantum systems. Bokulich argues that this quantum phenomenon is more deeply explained by appropriating concepts and models from classical periodic orbit theory, than it is by employing quantum mechanical models. This claim prompts the third section of the paper in which the structural aspect of the account is confronted with the objection that it lacks the ability to debar non-explanatory models such as those of Ptolemaic astronomy. The worry is that the way it is described all models exhibit structural properties of the target system, and so all equally satisfy the structural criterion, rendering it ineffective as a criterion for explanation. In order to evaluate this concern, I look at two reasonable approaches to measuring or assessing a model's structural information, and I argue that neither approach is ultimately satisfactory. The simplest approach of just measuring structure proves at worst impossible and at best arbitrary, and the comparative approach, while succeeding in debarring the Ptolemaic explanation, fails to find the highlyidealized model explanatory. The criterion either wrongly identifies all models as explanatory, or prefers models from fundamental theory. Either way, it cannot capture the way that highlyidealized models explain.

\section{Structural Model Explanations}

This section examines Bokulich's structural model account of explanation as laid out in (Bokulich, 2008) and incorporates the responses and clarifications made in (Bokulich, 2011, 
2012). Bokulich's account of explanation relies in part on work done by James Woodward, in particular the notion of depth he developed along with Christopher Hitchcock, which I go over first (Woodward \& Hitchcock, 2003a, 2003b). This notion is rather important for Bokulich as it forms the basis of the structural criterion of her account. I then show how her structural account aims to capture the explanatory ability of semiclassical models, classical models which can be used to approximate quantum systems. I do this by looking at the phenomenon of quantum wavefunction scarring and demonstrating how it satisfies the account's criteria. This shows both how a successful structural explanation is intended to proceed and motivate some of the intuitions we have about highly-idealized models being explanatory.

On Woodward's account, causality is framed in terms of counterfactuals rather than in terms of causal mechanisms or physical interaction (Woodward, 2003; Woodward \& Hitchcock, 2003b). Causal relationships, he claims, are out there in the world, but they can be well described in the reliable variable dependency relations of models. Explanation is the activity of gaining information about these causal relations by discovering through intervention which dependency relations are strongly invariant. The counterfactual dependency of these relations gives us important information that provides explanatory depth. This is information that answers what-ifthings-had-been-different questions, or w-questions. Thus, the range of questions that counterfactual dependence answers is related to the explanatory power of that causal relation. This is because it is important to see "what sort of difference it would have made for the explanandum if the factors cited in the explanans had been different in various possible ways" (Woodward, 2003, p. 11).

Bokulich rejects the causal approach and favours a structural interpretation of counterfactual information, or depth. In fact, she aims to give an account that can capture the explanatory ability of the highly-idealized, non-causal models that is not captured by Woodward's account. She has in mind the models of semiclassical mechanics. She claims that these models are not judged explanatory on a causal account because the entities involved (electron trajectories) are fictional and have no real causal power. As will be shown in more detail below, the morphologies (scarring patterns) of the quantum systems of interest correlate strongly with the particular periodic, or repeating, orbits of semiclassical mechanics, but the orbits cannot be said to cause the wavefunction distributions, even though there is a reliable dependency relation. Bokulich argues that none of the three main types of accounts of 
explanation (causal, covering law, and mechanistic) can capture the way semiclassical models explain quantum phenomena, and offers her own structural model explanation as a supplement. This account highlights the structural similarities between the real world system and the idealized or fictional model. Bokulich argues that structural model explanations are ones in which there is a pattern of counterfactual dependence among the variables of the model, which can be measured in terms of w-questions, and that this dependence is a consequence of the structural features of the target system (Bokulich, 2008, p. 145).

In developing her account, Bokulich draws on a suggestion made by Margaret Morrison that explanation has to do with structural dependencies (Morrison, 1999). Similar ideas have been developed by John Worrall, James Ladyman, and others (Esfeld \& Lam, 2008; French \& Ladyman, 2003; Ladyman, 1998; Worrall, 1989), but Bokulich's account does not draw heavily from these. Rather, Bokulich offers three general requirements for a structural model explanation, which I have paraphrased and enumerated as follows. The first criterion is E1, which states that the explanation makes reference to a scientific model, $M$. E1 specifies that the explanation is a model explanation and not a covering law or mechanistic explanation. The structural aspect of the structural model explanation comes from the second criterion E2, which says that $M$ is explanatory by showing how there is a pattern of counterfactual dependence of the relevant features of the target system on the structures represented in $M$. E2 is intended to determine which models are genuinely explanatory by ensuring that an explanatory model bears a close structural similarity to the counterfactual structure of the target system. This structural 'isomorphism', as she calls it, is given an quantitative measure in terms of w-questions (Bokulich, 2008, p. 145). The final criterion is E3, which states that there must be a justification that specifies the domain of the application of $M$. E3 is what she refers to as the justificatory step, intended to specify "where and to what extent the model can be treated as an adequate representation of the world" (Bokulich, 2008, p. 146).

The focus of this paper will be on the structural criterion E2, as I intend to show that structure cannot capture the way that highly-idealized models explain. There is a worry that all models exhibit a pattern of counterfactual dependency, and that therefore E2, satisfied by all models, is an idle wheel. Bokulich herself does not think that structure can distinguish explanatory from non-explanatory models, and instead relies on E3. But, if E2 is an idle wheel and E3 is little more than a judgment about what is an adequate representation in contemporary 
science, then the notion of explanation is unanalyzed - what is explanatory is what is considered explanatory by the current state of science. However, I think that there is a more promising avenue for structure: I argue that the criterion can in fact show preference for certain models over others when employed in a comparative approach. However, I also argue that this is unhelpful for Bokulich as the criterion shows preference for models of fundamental theory over those of highly-idealized models. I return to these approaches in Section 3, but now let us turn to the case study of structural model explanations in practice.

Bokulich applies her criteria for explanation to some cases of semiclassical mechanics as part of a larger project of reconceiving the intertheoretic division between the quantum and the classical. She argues that semiclassical mechanics can be genuinely explanatory of certain quantum systems even though they are either deemed non-explanatory or fall outside of the range of other accounts of explanation. The reason seems to be that the models of semiclassical mechanics are non-Galilean, or highly idealized. The distinction between Galilean and nonGalilean idealizations is one that was made popular by Ernan McMullin and it can help clarify the special nature of these models (McMullin, 1985). Very simply, if one can add detail to an idealized model and continually get closer to the real system, then this is what is known as a Galilean idealization. For instance, Galileo, in determining the rate of falling bodies, made use of balls rolling down incline planes that were assumed to be frictionless. This kind of idealization is harmless, and the same mathematical relation at which Galileo arrived can be modified to include friction to increase its accuracy. Even though they are not entirely accurate, models featuring these idealizations can be explanatory for McMullin. They represent the target system in a straightforward fashion and their use in explaining the system is justified in part by the fact that they approximately represent and can be "de-idealized". This process of de-idealizing is what ties the model to the real world ensures its approximate representation. Robert Batterman has described these models as having "controllable" idealizations, in that the idealizations of the system are justified theoretically (Batterman, 2005, p. 235). Idealizations that are non-Galilean on the other hand cannot be de-idealized to smoothly approach the real-world system. Many of these systems identified by Batterman have singular limits which preclude this possibility. These models then lack the approximate representation that is traditionally thought to justify their use in explanation. However, Batterman, Bokulich, and others argue that this does not preclude explanation. 
Models of semiclassical mechanics are highly idealized because it is not possible to recover the quantum models by removing approximations and de-idealizing. If the semiclassical models have explanatory power, it cannot be due to an underlying causal mechanism of which they are an approximate representation recoverable via de-idealization. Bokulich thinks that semiclassical models of quantum wavefunction scarring are precisely the kinds of structural explanations that Woodward's 2003 account cannot consider explanatory. This is why she allows that the justification of the application of the model to quantum phenomena (E3) can be topdown from theory, rather than bottom-up where it would be smoothly recovered in Galilean idealization. For semiclassical mechanics there is no smooth approximation, but there are topdown theories that can be used to model quantum features in classical terms.

Batterman was the first to argue that semiclassical appeals to classical structures in quantum phenomena at the asymptotic limit between the two is explanatorily important (Batterman, 1992, 2002b). Bokulich claims that structural explanations are actually quite popular in mechanics, where appeals to structural restrictions can account for certain aspects of systems. She argues that semiclassical mechanics can be an important interpretive and explanatory tool for certain quantum phenomena, specifically in the subfield of quantum chaos. Classical chaos is found in a great number of systems in which there is an extreme sensitivity to initial conditions, such that an immeasurably small difference in two initial conditions may result in an exponential divergence between them. Of course, this kind of extreme sensitivity to initial position and velocity has no part in quantum theory, but quantum models that also describe these systems are expected to exhibit something like chaos themselves. According to Bokulich and Batterman, one expects to find a correlate of classical chaos in quantum. Due to the agreement between quantum and classical predictions at the appropriate limit, there ought to be quantum systems that underlie classically chaotic systems as well (Batterman, 1992, pp. 51-52; Bokulich, 2008).

One of Bokulich's strongest examples is that of quantum wavefunction scarring in systems known as quantum billiards. These are systems where the wavefunction of a particle inside a stadium-shaped enclosure exhibits unusual patterns, which are called scars. Studies of these quantum billiard systems by means of semiclassical mechanics and cellular automata have revealed that there is an unusual accumulation of the wavefunction density along the trajectories that would be periodic orbits in a classical system (King, 2009). The strong correlation between the classical orbits and the observed quantum phenomenon makes these systems useful for 
studying the quantum systems underlying classical chaotic systems. Bokulich argues that these semiclassical approaches can be genuinely explanatory of the quantum scarring phenomenon.

Work on these stadium billiards was introduced by Leonid Bunimovich (Bunimovich, 1974). In the classical billiard systems, a stadium shaped enclosed space is inhabited by a freemoving particle whose trajectory is mapped. The boundary is defined by two semicircles connected by parallel lines, and the particle suffers specular reflections with the boundary. The shape of the enclosure has been since shown to be chaotic no matter how short its parallel segments are and to exhibit other interesting properties (Bleher, Ott, \& Grebogi, 1989; Bunimovich, 1979). One implication of these results is that a mapped trajectory of the particle generally displays an irregular pattern (Figure 1). This irregular pattern eventually leads to a uniform distribution of trajectories throughout the space.

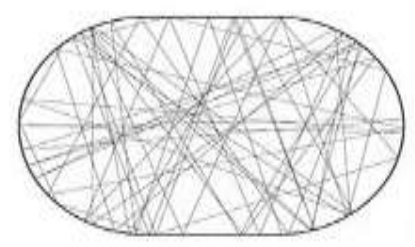

Figure 1 A typical example of a classical chaotic trajectory of a particle in a stadium shaped enclosure (Stöckmann, 2010).

However, the chaos of this system is intermittent, as there are certain special initial conditions that lead to periodic orbits in which the motion of the particle constantly repeats itself. There are certain starting positions and velocities that will not result in a uniformly distributed stadium, but exhibit a simple pattern of repeated motion. This pattern can occur in different shapes including a rectangle, a vee, and a bow tie, among others, and are called periodic orbits (Figure 2). 

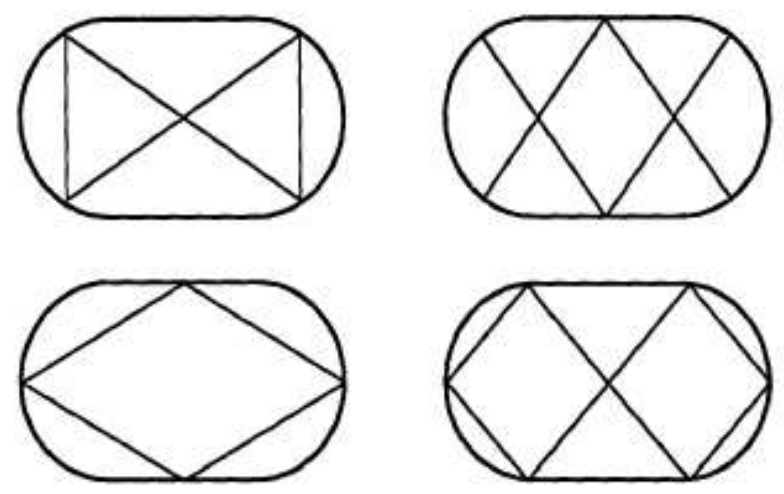

Figure 2 Examples of periodic orbits in a stadium enclosure (Heller, 1986).

These periodic, or bouncing ball, orbits exhibit a "stickiness" on nearby chaotic trajectories. The nearby trajectories, though not exactly periodic, exhibit quasi-regular behaviour for long periods of time. When mapped out, this stickiness results in an accumulation of trajectories in the near vicinity of the periodic orbits, due to the vanishingly small local Lyapunov exponent, which gives the strength of the scar (Dettman \& Georgiou, 2011). This property of stickiness is also exhibited in other shapes of dynamical billiards such as in drivebelt shapes, where the semicircles are of different sizes, and in various mushroom shapes, such as the one seen in Figure 3.

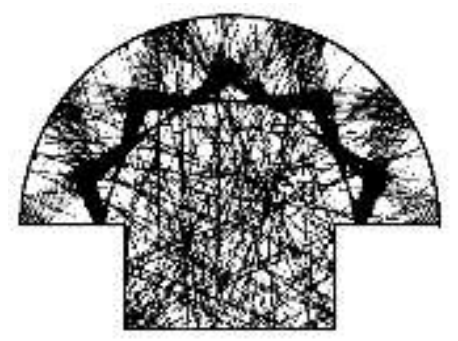

Figure 3 A mushroom shaped billiard system that clearly exhibits intermittent chaos. There is stickiness in the vicinity of the periodic orbits and a region of diffuse chaotic trajectories elsewhere (Dettman \& Georgiou, 2010).

What is very interesting about these systems is that because there are no trajectories in the quantum analogs of these systems, one would expect to be unable to distinguish the chaotic trajectories and the periodic orbits. Without orbit theory and sensitivity to position and velocity, there is no obvious reason to expect these particular strong patterns in the quantum wavefunction 
density. The interesting fact is that the quantum scarring phenomena actually converge on the classically stable periodic orbits. It is almost as if the quantum system is sensitive to which classical trajectories are periodic orbits and which are chaotic - a distinction that relies on the details of the particular trajectory.

The structural model explanation claim is that the phenomenon of quantum wavefunction scarring is best explained with the semiclassical model of the particle's behaviour. On the semiclassical account, the shape and size of the enclosure leads to certain periodic orbits being favoured and exhibiting stickiness on nearby trajectories. And so as one changes the shape, the allowed periodic orbits change in predictable ways, and the measured quantum wavefunction distribution also changes accordingly. The semiclassical model is highly idealized and nonrepresenting. The use of electron trajectories, which are false of the quantum system, is justified for Bokulich by means of Gutzwiller's periodic orbit theory, which is a method of approximating the density of quantum states from classical periodic trajectories. Gutzwiller's theory specifies how the behaviour of a Gaussian wavepacket $\varphi(x, 0)$ can serve as accurate solutions to the timedependent Schrödinger equation, and thus how the allowed classical periodic orbits correspond to the accumulation of wavefunction density (phase interference) observed as the scarring phenomenon (Heller, 1984). By considering the autocorrelation function of a Gaussian wavepacket, $\langle\varphi(t) \mid \varphi(0)\rangle$, on a phase space point associated with a periodic orbit, one can see an increase as the wavepacket overlaps with its initial state. The Fourrier transform of this function can be used to calculate the quantum spectrum. If the wavepacket is not initially on a phase space point associated with periodic orbit there will be no pattern of increases in the autocorrelation as it propogates, and hence no significant accumulation of the wavefunction in that region (Bokulich, 2008, pp. 128-129).

Bokulich does not argue that quantum mechanics cannot predict the scarring phenomenon. In the quantum analog, the scarring phenomena can be reproduced by means of the dynamics of the time-dependent Schrödinger equation with Dirichlet boundary conditions, so the function vanishes at the walls. Rather than a classical bouncing ball in a stadium, the model features a wave packet propagated through an infinite potential well with a stadium shaped boundary. In a similar manner to the simpler rectangular infinite well, one can show that the system will lead to ordered wavefunctions, and exhibit aphase interference pattern observed as the scarring phenomenon (Figure 4). As the system evolves, the wavefunction settles on a 
periodic solution. The scarring occurs when the probability amplitudes overlap in certain areas as the wavepacket propagates throughout the space and reflects off the boundary (King, 2009). ${ }^{1}$

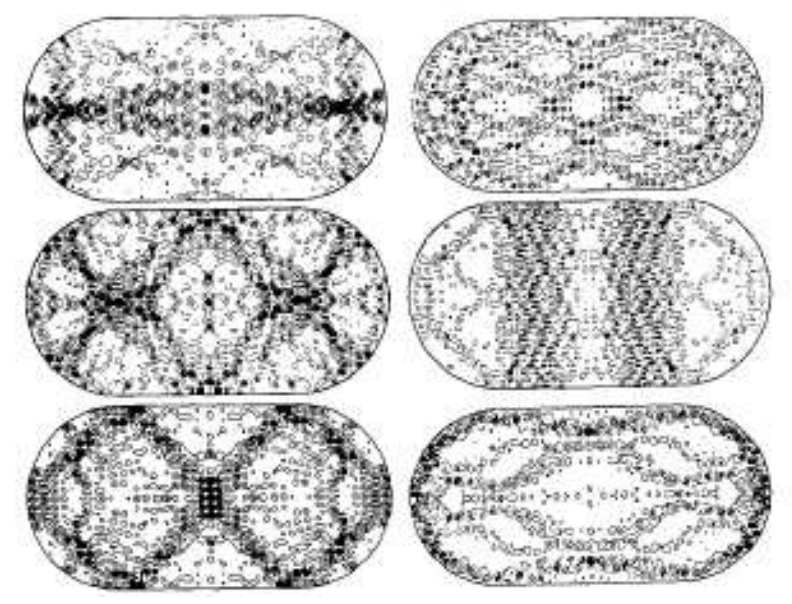

Figure 4. Amplitude contour maps of eigenstates that display a strong correspondence with the periodic orbits (Heller, 1986).

Bokulich is primarily interested in the explanatory potential of the semiclassical model. She argues that classical periodic orbits, though fictions - false of the quantum system - are explanatorily relevant to the phenomenon of quantum wavefunction scarring. By falsely assuming that the particle travels along a classical continuous space and time trajectory, one correctly expects to find certain scarring patterns in quantum billiard systems, which one would not expect prima facie in a quantum system. She argues that this example is a case of bona fide structural model explanation. This example is not an outlier case, but one of many in which Bokulich reaches the same conclusion about explanation; including the conductance peaks of ballistic quantum dots, the orbits of Bohr's model of the atom, and the resonance peaks of the Rydberg electrons (Bokulich, 2008).

For Bokulich, these examples suggest that there is a "dynamical structural continuity" between the classical and quantum theories. Because of this she argues that semiclassical

\footnotetext{
${ }^{1}$ For more information about the quantum models, simulations of the scarring phenomen, and ergodic and unique ergodic properties of classical and quantum billiards, see (Dettman \& Georgiou, 2010; Gutzwiller, 1990; Heller, 1984, 1986; Kaplan \& Heller, 1999; King, 2009; McDonald \& Kaufman, 1979; Tao, 2007; Tomsovic \& Heller,
} 1993) 
fictions, in this case the classical periodic orbit theory applied to a quantum particle, can serve to give counterfactual information about the quantum system. The closed orbits are not real, in the sense that the particle is not actually travelling in a classically defined orbit with position and velocity. Bokulich does not want to argue that the trajectories are real, but rather that they are a special kind of fiction that is also explanatory: "These closed and periodic classical orbits can be said to explain features of the spectral resonances and scarring insofar as they provide a semiclassical model of these phenomena" (Bokulich, 2008, p. 140). The dependence of the scarring phenomenon on the classical orbits conveys physical insight, or structural information, on the underlying quantum dynamics.

Of course, in order for Bokulich to claim that there is a genuine explanation here, the semiclassical model must satisfy her three criteria E1-3. And it can be quickly shown that it does. The explanation makes reference to a scientific model, viz. Gutzwiller's periodic orbit theory, and so it satisfies E1. The semiclassical model exhibits a strong counterfactual dependence of the probability density in the billiard systems on the particular periodic orbits. It satisfies E2, the structural criterion, because one is able to say how the wavefunction morphology inside the stadium would change if the periodic orbit had been different, or if the shape of the stadium had been changed. This semiclassical explanation is also justified in being applied to this domain (E3) because Gutzwiller's periodic orbit theory specifies how to model features of quantum dynamics with classical trajectories - how to get real-world information from the information in the model. So for Bokulich, this semiclassical model qualifies as explanatory.

As we have seen, Bokulich does not claim that quantum mechanics alone cannot predict these scarring phenomena. Rather, her claim is that its explanations are deficient because they do not provide as much counterfactual information about the system, which gives us physical insight into the system and grants understanding. In order to get a more concrete sense of the counterfactual information a model gives about the system, she makes use of w-questions and Woodward and Hitchcock's notion of explanatory depth, mentioned earlier. The more wquestions a model answers, the more structural information it gives, the deeper the explanation it provides (Bokulich, 2008, p. 152). Bokulich argues not only that the semiclassical models are explanatory, but that the semiclassical models actually provide deeper explanations than the fully quantum ones: "More importantly, the semiclassical models provide more information about the structure of the quantum dynamics than do the fully quantum calculations. That is, the 
semiclassical model allows one to answer a wider variety of w-questions, about how the system would behave if certain parameters were changed..." (Bokulich, 2008, p. 154). Reference to the classical structures is eliminable in that one can make simulations that exhibit scarring phenomena from a quantum model. Nonetheless, Bokulich argues that "without knowledge of the classical orbits, our understanding of the quantum spectra and wavefunction morphologies is incomplete" (Bokulich, 2008, p. 154).

For Woodward and Hitchcock, the range of w-questions that a model can reliably answer about a phenomenon is directly related to the model's range of invariance. Brad Weslake has identified many distinct measures of invariance in their account (Weslake, 2010). For instance, a model can be more invariant if it is more accurate within a certain range; invariant under a wider, or more continuous range of interventions; invariant under a wider range of different ways the interventions may be performed; or under a wider range of background conditions. "What they have in common is that they provide the resources to describe a greater range of true counterfactuals concerning the possible changes to the system in question - that is, to answer more w-questions..." (Weslake, 2010, p. 278). All of these different kinds of invariance are relevant to the structural information Bokulich is looking for in E2.

Bokulich claims that the semiclassical model of wavefunction scarring gives counterfactual information about the quantum system, and further that "there can be situations in which less fundamental theories can provide deeper explanations than more fundamental theories" (Bokulich, 2008, p. 153). Given that there are local models framed entirely in quantum terms that can predict these scarring phenomena, if Bokulich wants to argue that the semiclassical model provides deeper explanations than the fully quantum one, then she has to show that the semiclassical model can answer a wider range of w-questions, in the full sense described above.

\section{Two Approaches for Assessing Structure}

I have shown how Bokulich's account aims to capture the way highly-idealized models like those of semiclassical mechanics explain, and I now turn to examine some worries about the claim. As stated above, the worry is that all models satisfy E2, and when E3 has to do all the heavy lifting, it is not enough for an account of explanation. In order to make the best case for a structural criterion, I present two possible ways of assessing structure: a direct and 
straightforward measure of the number of answers to w-questions (or w-answers) with a certain threshold for explanation, and an indirect measure for comparing the different classes of wanswers that two competing models provide. I aim to show that both avenues for assessing structure are unsatisfactory. The first approach proves impossible and leaves E2 an idle wheel. The second, when it is possible, does not side in favour of the highly-idealized model, which it was intended to do. Further, I suggest that this is not something particular to w-answers but applies generally to any similar measure for structure. Now, let me first turn to the original worry about structure, before presenting the more promising view.

In a review of (Bokulich, 2008), Belot and Jansson express a concern that once the account of structural model explanations allows for such fictions as classical trajectories in quantum systems, it will be unable to reject models that are widely considered non-explanatory, such as the models of Ptolemaic astronomy. They ask, "what is to stop you from viewing the Ptolemaic model of the solar system as giving an adequate structural model explanation of this phenomenon? Indeed, an appeal to the Ptolemaic model on this question would appear to satisfy all four requirements for a structural model explanation” (Belot \& Jansson, 2010, pp. 82, 83). The worry is that once she opens the door up to explanatory fictions her criteria are not strong enough to debar non-explanatory fictions, such as planetary epicycles. Bokulich (2012) is explicit in wanting to allow for the fictitious electron trajectories in quantum billiard systems, but not the fictitious epicycles of Ptolemaic astronomy. As is well known, the Ptolemaic model of the solar system makes use of epicycles, on which there is a strong counterfactual dependence of the apparent retrograde motion of the planets across the night sky as seen from Earth. At first glance, Belot and Jansson are right to worry that epicycles and electron trajectories both satisfy E2, but Bokulich argues that her account is capable of admitting one and not the other. Let us first see whether the Ptolemaic explanation for planetary motion satisfies her three criteria for a good explanation.

The Ptolemaic explanation satisfies E1, insofar as it references the geocentric model of the solar system. The model is also counterfactually reliable under certain conditions. The Ptolemaic system has trigonometric tables of chords used for calculations, and these give us counterfactual information about the visible solar system. And so it also seems to satisfy E2. It is only on the third criterion E3 that the Ptolemaic models will be debarred according to Bokulich. The geocentric model and its epicycles are not at all adequate representations of the real 
structure of solar system, and so not deemed relevant to the explanation of planetary motion by the contemporary state of science (Bokulich, 2012, p. 735). This is undoubtedly true but it is irrelevant to the success or failure of a structural criterion.

For a structural model explanation, the explanatory power of the model ought to come from the model's ability to reliably give counterfactual information about the behaviour of the system. Bokulich's account requires that the "model must explain the explanandum by showing how there is a pattern of counterfactual dependence of the relevant features of the target system on the structures represented in the model" (Bokulich, 2008, p. 146). Unlike how it is framed in Woodward and Hitchcock, for Bokulich there is no need to insist that the counterfactual dependency is representative of causal relations. Rather, she claims what is being exhibited is the structural dynamics of the system. In order to most accurately assess the satisfaction of E2, one needs to actually measure the w-answers that a model can provide.

Unfortunately, obtaining a measure of the number of w-questions a certain model can answer is not straightforward. Let us examine one approach, which is simply to count the number of w-questions answered by a model and if the number is sufficiently large then the model is explanatory. However, two problems with this immediately arise. First, the Ptolemaic system, for instance, has methods of calculating the positions of the bodies of the solar system for any given day, for any place on Earth, including not just positions in the night sky, but eclipses, solstices, equinoxes, and so on. Importantly, these bodies have cycles and epicycles that are geometrically continuous. This means that the model provides an infinite number of wanswers, as there are an infinite number of points on the lines of the spherical trigonometry. And so the number of w-questions answered by even a non-explanatory model is infinite. One could make a stronger case for this approach by articulating a method which looks at the size of the parameter space, the domain of values allowed by the model. This might allow one to get more varied information about the amount of counterfactual information a model provides. This is an interesting approach, but I cannot explore it in detail here. However, even if one were equipped with a good quantitative measure of the structural information a model provides, the second problem remains: there is no principled way of determining exactly how many w-answers or what size of measure would count as "sufficient" for explanation. Without some larger framework for determining exactly the minimum size, a proposed threshold seems arbitrary. 
And so this approach fails, as all models would equally satisfy E2, and thus the entirety of judging explanation falls on E1 and E3, which without E2, amounts to nothing more than reflecting current judgments about explanation. In this case, there is no analysis of explanation and no reason to think of these as structural explanations. However, I think there is a more promising comparative approach. While Bokulich does not explicitly frame w-questions in a comparative way, I suggest that the Ptolemaic model does answer fewer w-questions than, for example, the Newtonian model, and it can be debarred in that fashion. As we have seen, a quantitative method for counting w-answers is not possible, and so a simple quantitative comparison cannot be made either. Bokulich suggests examining the classes of w-questions, and I believe that there is a sense in which an intuitive comparison of the classes of w-questions is possible. Consider a relative formulation of the structural criterion, which states that $M 1$ provides deeper explanations than $M 2$ if $M 1$ if it gives more counterfactual information than $M 2$, which is given in terms of classes of $\mathrm{w}$-questions.

Because there is a lot of overlap in the information one gets from the Ptolemaic and Newtonian models of the solar system, an argument could be made that the Ptolemaic model has a narrower scope, which is to say that the Newtonian model can give all the w-answers that the Ptolemaic model provides, but also a lot of additional w-answers as well. For instance, one can get reliable w-answers about how the orbits would be different if the planets or the Sun had different masses, or if the planets were different distances from the Sun, and so on. But as Woodward and Hitchcock were careful to point out, there is more to explanatory depth than scope. In this case, the Newtonian model answers more different kinds of w-questions more accurately. It gives accurate, stable, and robust counterfactual information. The Ptolemaic model does not fare well in this kind of comparison, so perhaps a comparative structural criterion can be capable of distinguishing explanatory from non-explanatory models. This could provide a defense for this structural approach from criticisms like those raised by Belot and Jansson.

If this comparative framework works for Ptolemaic astronomy, is it also capable of showing that, as Bokulich argues, the semiclassical model provides a deeper explanation of the scarring phenomenon than the fully quantum model featuring the Schrödinger equation? Well, when one returns to the semiclassical model and attempts to compare the w-answers with those provided by the local quantum model, the comparison does not seem to lead to the conclusion that the highly-idealized model provides deeper explanations. 
The semiclassical model can give counterfactual information about the distribution of probability densities in the enclosure in straightforward way. There is a certain range of questions that can be answered about the dependence of the wavefunction morphology on the classical orbits. There is a striking correlation between the trajectories predicted by the classical theory and the observed phenomenon. The semiclassical model is indifferent to the details and particulars of the quantum dynamics and allows certain features like scarring phenomena to be highlighted. Information about why particular scarring patterns, as seen in Figure 4, occur is given by the semiclassical model, so the argument would go, because it is easily capable of accounting for the chaotic and the particular periodic trajectories, and can show how the quantum scarring would change if things (the periodic orbits in Figure 3) had been different. It does seem that here too, "rather than obscuring the genuine mechanisms at work, this idealization actually brings them into focus" (Batterman, 1992, p. 64). So it seems plausible that there could be a class of w-questions that are more deeply, or at least more intuitively, answered by the highly-idealized model.

Bokulich argues that the non-fundamental model can provide deeper explanations. But the semiclassical model does not does not exclusively account for the scarring phenomena. The Schrödinger equation can be used in a quantum model to achieve the results that are obtained in the semiclassical model. As Bokulich freely admits: "one can "deduce" the phenomenon of wavefunction scarring by numerically solving the Schrödinger equation," the problem, she argues, is that "such an explanation fails to provide adequate understanding of this phenomenon" (Bokulich, 2008, p. 151). Understanding, for Bokulich, is given by the physical insight, or information in terms of w-questions that a model answers. And so, this means that the nonfundamental model provides more w-answers. However, Weslake, points out that the w-question notion of explanatory depth favours more fundamental generalizations: "The fundamental laws are those generalizations that are maximally accurate, robust, continuous, stabile, insensitive, and portable" (Weslake, 2010, p. 278). This is what many assume to be the case and is what Woodward and Hitchcock assume when they explored the notions of depth and counterfactual information (Woodward \& Hitchcock, 2003b).

It is worth unpacking this assumption. What is crucial is that the quantum model can account for the same scarring phenomena as the semiclassical model. Of course, the quantum model cannot answer specific, classically-framed w-questions about trajectories or Lyapunov 
exponents, because the concepts are unavailable to the quantum model. But, while the fundamental model loses w-questions in terms of trajectories, it gains "corresponding" wanswers framed in terms of wave packet propagation. The quantum model is capable of giving counterfactual information (w-answers) about the morphology of the scarring patterns.

But the local quantum model is also able to provide very deep explanations of these phenomena, because as a model of a fundamental theory it can provide so much counterfactual information. This seems to give the same comparative relation between Ptolemaic and Newtonian models of the solar system. Though this comparative approach is not strictly quantitative, intuitively, the quantum model can be seen to give at least as many w-answers as the semiclassical model, given that whenever the semiclassical model can give information about wavefunction morphology, so can the quantum model. However, the quantum model can also provide additional counterfactual information about a variety of w-questions that can be answered by the quantum dynamics of the system in terms unavailable to the semiclassical model. While the explanations themselves may differ, the phenomena explained by the semiclassical model are a proper subset of the phenomena explained by the local quantum model. The semiclassical method is an approximation, which is poor in certain conditions and fails in others. While a quantitative analysis of the closeness of the semiclassical approximation is more properly suited to a physics paper, it is clear the equations of the fundamental model that are more accurate and more strongly invariant, in the many senses outlined above. An approximation can be very useful, but it does not contain more information.

It is certainly possible that one not choose w-questions and this notion of explanatory depth to form the basis of a measure of structure. Others have attempted to capture explanatory depth by other means (Schupbach \& Sprenger, 2011; Strevens, 2008; Weslake, 2010), but few if any have offered concrete measures of structure. One of the aims of offering a structural account is to preserve autonomy of higher level explanations, and to allow some highly-idealized and non-causal models, such as those of semiclassical mechanics, to be considered explanatory. I admit that it is possible that an account of explanatory depth might be able to preserve autonomy, but any approach to quantifying structure, whether w-questions or not, will meet with the difficulties I have outlined.

However, this comparative approach has an interesting corollary. If there were a domain of phenomena in which the model of the more fundamental theory could not derive the desired 
results, or reproduce the phenomena, then the best available explanation would be given by the model of the less fundamental theory - which is to say that hope is not lost for this approach in capturing the way some highly-idealized models explain. In this particular case however, Bokulich admits that a quantum model can account for the scarring phenomena described by the semiclassical model. And so it turns out that even though the classical trajectories can answer interesting $\mathrm{w}$-questions about the particular morphologies of the wavefunction scarring, models from the more fundamental theory will always win out in terms of w-questions when they can account for the same phenomena, because they can answer at least as many classes of wquestions. This does not mean that depth is incompatible with autonomy. Weslake provides a different abstractive account of depth, which he argues is more promising for preserving autonomy (Weslake, 2010). An assessment of Weslake's arguments or of the possibility of incorporating different notions of depth into a structural account, fall outside the scope of this paper.

Woodward might be able to accept that these highly-idealized models are explanatory, but less explanatory than models of more fundamental theories that offer much deeper explanations, as long as they satisfy his criteria for explanation by exhibiting a strong enough degree of invariance under a range of interventions. However, this will not work for Bokulich in this case, because it will not favour the models of semiclassical mechanics over those of quantum mechanics because they are both capable of accounting for the scarring phenomena. It is important for Bokulich, not only that semiclassical models be explanatory, but that they actually provide deeper explanations of some quantum phenomena than the fully quantum explanations.

While this measure of structure sides in favour of models of more fundamental theories when they can predict the same phenomenon, when they cannot, an intuitive sense of which model answers more classes of w-questions does not seem to have any bearing on the explanatory depth of one model or the other. For example, if one compares a semiclassical model with a Ptolemaic one, regardless of how the w-answer balance tips, the structural criterion still has no real bearing on whether the models of semiclassical mechanics are themselves explanatory. When there is no overlap in the domain of the models, the comparison is not helpful. I contend that even if there were a quantitative way to measure the structural similarity using something other than classes of w-questions, these problems remain. Imagine it were possible to give a compressed scalar rating of all the complex representations of structural 
similarity, given by a complicated process of calculations and perhaps insights from measure theory. Now suppose that the Ptolemaic model of the solar system was given a rating of 4 and the semiclassical model of quantum wavefunction scarring a generous 8. Even though it received a higher ranking than the Ptolemaic model, it is still reasonable to ask "does the semiclassical model explain quantum wavefunction scarring?" And so it does not seem that there can be any way that such a comparative framework can provide a general criterion for explanation. It is only when both models can reproduce the same phenomenon that a meaningful comparison can be made, but when it can be made, it does not favour the highly-idealized model.

The main worry for a structural criterion for explanation is that some measure of structural similarity can be given to almost any model, no matter how inaccurately it represents, or how little structural information it provides. And so if one wishes to debar the worst of these then either a threshold must be drawn, or a comparison must be made. For these semiclassical models, a threshold proves unworkable. The comparison, when possible, sides in the favour of the models of the more fundamental theory and not the highly-idealized model. Further, this comparison is only helpful for models with overlapping domains, and leaves unanswered the question of whether a particular model explains its target phenomenon. It does not seem possible to conclude that the semiclassical model provides deeper explanations.

\section{Conclusion and Discussion}

Bokulich has taken bold steps forward in arguing that w-questions can be used to measure structural similarity. However, this measure proves rather difficult to determine. Neither of the two approaches I outlined is capable of concluding that the highly-idealized semiclassical model is explanatory. Even non-explanatory models provide an infinite number of w-answers, and even when employing measure theory a given threshold seems arbitrary. When comparing, one finds that a fundamental model wins out in terms of classes of w-answers. Either way, the account does not capture the way that highly-idealized models explain.

Because the contribution of E2 is negligible to whether a highly-idealized model is explanatory, the entirety of judging explanation falls on E1 and E3. Indeed, Bokulich herself does not appeal to structure in order to debar the Ptolemaic explanation, rather she argues that the model fails to be explanatory because it simply does not qualify as an adequate fictional 
representation of the solar system in contemporary science (it does not satisfy E3). The relevance relation set by the contemporary state of science has precluded explanations of planetary motion featuring epicycles, barometers causing storms, and shadows causing flagpole heights, but not necessarily the models of semiclassical mechanics. Semiclassical mechanics is still a fruitful research avenue, and it is intuitively powerful. It allows us to grasp and picture systems that we would otherwise not be able to picture, and frame them in familiar terms. And quite astonishingly, it can give us simple and reliable counterfactual information about certain quantum systems. Semiclassical mechanics is certainly more relevant to quantum phenomena than Ptolemaic epicycles are to planetary motion, because its models are heuristically valuable in providing frameworks for investigating and calculating quantum systems. However, this is not sufficient for them to be explanatory.

E3 only reflects our current judgments about whether a model is explanatory and does not make any claims about why the model ought to be considered explanatory. E3's descriptive nature takes away the normative aspect that an account of explanation ought to have, and has traditionally aimed for. I have other worries about E3 that are outside the scope of this paper, but my main worry is not that this criterion itself is problematic. Rather, it is that E3 has to play such a strong role in judgments about which models are explanatory in light of what I have shown about E2. I do not think that E1 and E3 alone can provide an adequate account of how highlyidealized models explain.

Highly-idealized models are common in science and as other have argued (Batterman, 2002a; Batterman \& Rice, 2014; Rice, 2012, 2013; Wayne, 2011), there is reason to consider them explanatory. Developing an account of explanation that more accurately reflects the explanatory practices science of is a next major step in the philosophy of science. Bokulich tries to do so by providing a quantitative measure for a structural criterion but it ends up not working in her favour. She attempts to preserve the autonomy of semiclassical, and perhaps other highlyidealized explanations, but her notion of structure is cashed out in a measure of explanatory depth that prefers more fundamental generalizations with strongly invariant mechanical laws. I have not argued that no notion of structure can preserve autonomy, but perhaps this is the wrong way to view the problem. Perhaps the best way to preserve autonomy is not by developing a structural account, but in exploring alternative approaches to explanatory depth. 


\section{References:}

Batterman, Robert W. (1992). Quantum Chaos and Semiclassical Mechanics. Proceedings of the Biennial Meetings of the Philosophy of Science Association, 1992(2), 15.

Batterman, Robert W. (2002a). Asymptotics and the Role of Minimal Models. British Journal for the Philosophy of Science, 53, 17.

Batterman, Robert W. (2002b). The Devil in the Details. Oxford: Oxford University Press,.

Batterman, Robert W. (2005). Critical Phenomena and Breaking Drops: Infinite Idealizations in Physics. Studies in History and Philosophy of Modern Physics, 36, 225-244.

Batterman, Robert W., \& Rice, Collin C. (2014). Minimal Model Explanations. Philosophy of Science, 81(3), 349-376. doi: 10.1086/676677

Belot, Gordon, \& Jansson, Lina. (2010). Review of Reexamining the Quantum-Clasical Relation: Beyond Reductionism and Pluralism, by A. Bokulich. Studies in History and Philosophy of Modern Physics, 41, 3.

Bleher, S., Ott, E., \& Grebogi, C. (1989). Routes to chaotic scattering. Physical Review Letters, 63(9), 919-922.

Bokulich, Alisa. (2008). Reexamining the Quantum-Classical Relation: Beyond Reductionsm and Pluralism New York: Cambridge University Press.

Bokulich, Alisa. (2011). How Scientific Models Can Explain. Synthese, 180(1), 13.

Bokulich, Alisa. (2012). Distinguishing Explanatory from Nonexplanatory Fictions. Philosophy of Science, 79(5), 725-737.

Bunimovich, Leonid. (1974). The ergodic properties of certain billiards. Functional Analysis and its Applications, 8, 73-74.

Bunimovich, Leonid. (1979). On the Ergodic Properties of Nowhere Dispersing Billiards. Communications in Mathematical Physics, 65, 295-312.

Cartwright, Nancy. (1983). How the Laws of Physics Lie. Oxford: Oxford University Press.

Craver, Carl. (2006). Physical Law and Mechanistic Explanation in the Hodgkin and Huxley Model of the Action Potential. Philosophy of Science, 75(5), 11.

Dettman, Carl P., \& Georgiou, Orestes. (2010). Open Intermittent Billiards: A dynamical window. from http://iopscience.iop.org/1751-8121/labtalk-article/46000

Dettman, Carl P., \& Georgiou, Orestes. (2011). Open Mushrooms: Stickiness Revisited. Journal of Physics: Mathematical and Theoretical, 44, 30pp.

Esfeld, Michael, \& Lam, Vincent. (2008). Moderate structural realism about space-time. Synthese, 160, 27-46.

French, S., \& Ladyman, James. (2003). Remodelling structural realism: Quantum physics and the metaphysics of structure. Synthese, 136, 31-56.

Gutzwiller, Martin C. (1990). Chaos in classical and quantum mechanics. New York: Springer Verlag.

Heller, E. J. (1984). Bound-State Eigenfunctions of Classically Chaotic Hamiltonian Systems: Scars of Periodic Orbits. Physical Review Letters, 53(16), 1515-1518.

Heller, E. J. (1986). Qualitative properties of eigenfunctions of classically chaotic Hamiltonian Quantum Chaos and Statistical Nuclear Physics, 263, 162 - 181.

Hempel, Carl G., \& Oppenheim, Paul. (1948). Studies in the Logic of Explanation. Philosophy of Science, 15(2), 40.

Kaplan, L., \& Heller, E. J. (1999). Measuring scars of periodic orbits. Physical Review E, 59(6), 6609-6628. 
King, Chris. (2009). Exploring Quantum, Classical and Semiclassical Chaos in the Stadium Billiard. Quanta, 3(1), 16-31.

Ladyman, James. (1998). What is structural realism? Studies in History and Philosophy of Modern Science, 29, 409-424.

McDonald, Steven W., \& Kaufman, Allan N. (1979). Spectrum and Eigenfunctions for a Hamiltonian with Stochastic Trajectories. Physical Review Letters, 42(18), 1189-1191.

McMullin, Ernan. (1985). Galilean Idealization. Studies in History and Philosophy of Science, 16(3), 26.

Morrison, Margaret. (1999). Models as Autonomous Agents. In M. Morrison \& M. Morgan (Eds.), Models as Mediators: Perspectives on Natural and Social Science (pp. 38 - 65 ). Cambridge: Cumbridge University Press.

Rice, Collin. (2012). Optimality explanations: a plea for an alternative approach. Biology and Philosophy, 27, 18.

Rice, Collin. (2013). Moving Beyond Causes: Optimality Models and Scientific Explanation. Noûs, 49(2).

Schupbach, Jonah, \& Sprenger, Jan. (2011). The Logic of Explanatory Power. Philosophy of Science, 78(1), 105-127.

Stöckmann, Hans-Jürgen (2010). Stoe Billiards. In stoe_billiards.jpeg (Ed.). Sholarpedia.

Strevens, Michael. (2008). Depth: An Account of Scientific Explanation. Harvard, MA: Harvard University Press.

Tao, Terrence. (2007). Open question: scarring for the Bunimovich stadium. Retrieved from http://terrytao.wordpress.com/2007/03/28/open-question-scarring-for-the-bunimovichstadium/

Tomsovic, Steven, \& Heller, Eric J. (1993). Long-time semiclassical dynamics of chaos: The stadium billiard. Physical Review E, 47(1), 282-299.

Wayne, Andrew. (2011). Extending the Scope of Explanatory idealization. Philosophy of Science, 78(5), 11.

Weslake, Brad. (2010). Explanatory Depth. Philosophy of Science, 77(2), 273-294.

Woodward, James. (2003). Making Things Happen: A Theory of Causal Explanation. Oxford: Oxford University Press.

Woodward, James, \& Hitchcock, Christopher. (2003a). Explanatory Generalizations, part I: A Counterfactual Account. Nous, 37(1), 1-24.

Woodward, James, \& Hitchcock, Christopher. (2003b). Explanatory Generalizations, Part II: Plumbing Explanatory Depth. Nous, 37(2), 181-199.

Worrall, John. (1989). Structural Realism: The Best of Both Worlds? Dialectica, 43(1-2). 